\title{
The Role of the Humanities and Social Sciences in Nanotechnology Research and Development
}

\author{
Mette Ebbesen
}

Published online: 3 December 2008

(C) Springer Science + Business Media B.V. 2008

\section{Erratum to: Nanoethics \\ DOI $10.1007 / \mathbf{s} 11569-008-0033-z$}

\section{Retraction}

In the paper "The role of the humanities and social sciences in nanotechnology research and development" by Mette Ebbesen, in this journal, volume 2, number 1, pages 1-14, parts of the section "Potential strategy for incorporating the humanities and social sciences into the research process of nanotechnology" are identical with

The online version of the original article can be found at http:// dx.doi.org/10.1007/s11569-008-0033-z of the article with published errors.

\section{Ebbesen $(\square)$}

Centre for Bioethics and Nanoethics,

University of Aarhus,

Taasingegade 3, Building 1443,

8000 Aarhus C, Denmark

e-mail: meb@teo.au.dk

\section{Ebbesen}

Interdisciplinary Nanoscience Center (iNANO),

University of Aarhus,

Ny Munkegade,

8000 Aarhus C, Denmark material in David H. Guston and Daniel Sarewitz, "Real-time technology assessment", Technology in Society, volume 24, 2002, pages 93-109. Parts of the section "Integrating societal concerns into nanotechnology" are identical with material in Erik Fisher and Roop L. Mahajan, "Contradictory intent? US federal legislation in integrating societal concerns into nanotechnology research and development", Science and Public Policy, volume 33, 2006, pages 5-16. Both papers were extensively referenced throughout the relevant sections and there was no suggestion by Ebbesen that the ideas discussed were her own. However there was no indication that substantial parts of the sections mentioned above were identical with material in those cited papers. The author had no intention to mislead and sincerely regrets having given the impression that the words were her own. All reasonable steps will be taken to minimise the risk of this happening again and I sincerely apologise to the authors, David Guston and Daniel Sarewitz, and Erik Fisher and Roop L. Mahajan, to their respective publishers, Elsevier Science Ltd and Beech Tree Publishing, and to the readers of NanoEthics for publishing this as an original paper.

John Weckert

Editor-in-Chief 